\title{
Robotic Emotional Expression Generation Based on Mood Transition and Personality Model
}

\author{
Meng-Ju Han, Chia-How Lin, and Kai-Tai Song, Member, IEEE
}

\begin{abstract}
This paper presents a method of mood transition design of a robot for autonomous emotional interaction with humans. A 2-D emotional model is proposed to combine robot emotion, mood, and personality in order to generate emotional expressions. In this design, the robot personality is programmed by adjusting the factors of the five factor model proposed by psychologists. From Big Five personality traits, the influence factors of robot mood transition are determined. Furthermore, a method to fuse basic robotic emotional behaviors is proposed in order to manifest robotic emotional states via continuous facial expressions. An artificial face on a screen is a way to provide a robot with a humanlike appearance, which might be useful for human-robot interaction. An artificial face simulator has been implemented to show the effectiveness of the proposed methods. Questionnaire surveys have been carried out to evaluate the effectiveness of the proposed method by observing robotic responses to a user's emotional expressions. Preliminary experimental results on a robotic head show that the proposed mood state transition scheme appropriately responds to a user's emotional changes in a continuous manner.
\end{abstract}

Index Terms-Emotional model, facial expression generation, facial expression recognition, robotic behavior fusion, robotic emotional interactions, robotic mood state transition.

\section{INTRODUCTION}

$\mathbf{T}$ HE DEVELOPMENT of domestic and service robots has gained increasing attention in recent years. The market of service robots is forecasted to grow fast in the future. One of the most interesting features of intelligent service robots is their human-centered functions. Intelligent interaction with a user is a key feature for service robots in health-care, companion, and entertainment applications. For a robot to engage in friendly interaction, the function of emotional expression will play an important role in many real-life application scenarios. However, it is known that to make a robot behave humanlike emotional expressions is still a challenge in robot design.

Methodologies for developing emotional robotic behaviors have drawn much attention in the robotic research community [1]. Breazeal et al. [2] presented the sociable robot Leonardo, which has an expressive face capable of near-human-level

Manuscript received December 14, 2011; revised May 30, 2012 and August 28, 2012; accepted November 5, 2012. Date of publication December 11, 2012; date of current version July 15, 2013. This work was supported in part by the Ministry of Economic Affairs under Grant 97-EC17-A-04-S1-054 and in part by the National Science Council, Taiwan, under Grant NSC-96-2628-E-009-162-MY3. This paper was recommended by Editor L. Shao.

The authors are with the Department of Electrical and Computer Engineering, National Chiao Tung University, Hsinchu 300, Taiwan (e-mail: menlo. ece92g@nctu.edu.tw; jotarun.ece87@nctu.edu.tw; ktsong@mail.nctu.edu.tw).

Color versions of one or more of the figures in this paper are available online at http://ieeexplore.iee.org.

Digital Object Identifier 10.1109/TSMCB.2012.2228851 expression and possesses a binocular vision system to recognize human facial features. The humanoid robot Nexi [3] demonstrated a wide range of facial expressions to communicate with people. Wu et al. [4] explored the process of selfguided learning of realistic facial expression by a robotic head. Mavridis et al. [5], [6] developed the Arabic-language conversational android robot; it can become an exciting educational or persuasive robot in practical use. Hashimoto et al. [7], [8] developed a reception robot, SAYA, to realize realistic speaking and natural interactive behaviors with six typical facial expressions. In [9], a singer robot, EveR-2, is able to acquire visual and speech information while expressing facial emotion during performing robotic singing. For some application scenarios such as persuasive robotics [10] or longer term human-robot interaction [11], interactive facial expression has been demonstrated to be very useful.

There have been increasing interests in the study of robotic emotion generation schemes in order to give a robot more humanlike behaviors. Reported approaches to emotional robot design often adopted results from psychology in order to design robot behaviors to mimic human beings. Miwa et al. proposed a mental model to build the robotic emotional state from external sensory inputs [12], [13]. Duhaut [14] presented a computational model which includes emotion and personality in the robotic behaviors. The Traits, Attributes, Moods, and Emotions framework proposed by Moshkina et al. gives a model of time-varying affective response for humanoid robots [15]. Itoh et al. [16] proposed an emotion generation model which can assess the robot's individuality and internal state through mood transitions. Their experiments showed that the robot could provide more humanlike communications to users based on the emotional model. Banik et al. [17] demonstrated an emotion-based task sharing approach to a cooperative multiagent robotic system. Their approach can give a robot a kind of personality through the accumulation of past emotional experience. Park et al. [18] developed a hybrid emotion generation architecture. They proposed a robot personality model based on human personality factors to generate robotic interactions. Kim et al. [19] utilized the probability-based computational algorithm to develop the cognitive appraisal theory for designing artificial emotion generation systems. Their method was applied to a sample of interactive tasks and led to a more positive human-robot interaction experience. In order to allow a robot to express complex emotion, Lee et al. [20] proposed a general behavior generation procedure for emotional robots. It features behavior combination functions to express complex and gradational emotions. In the authors' previous work [21], a design of autonomous robotic facial expression generation is presented. 


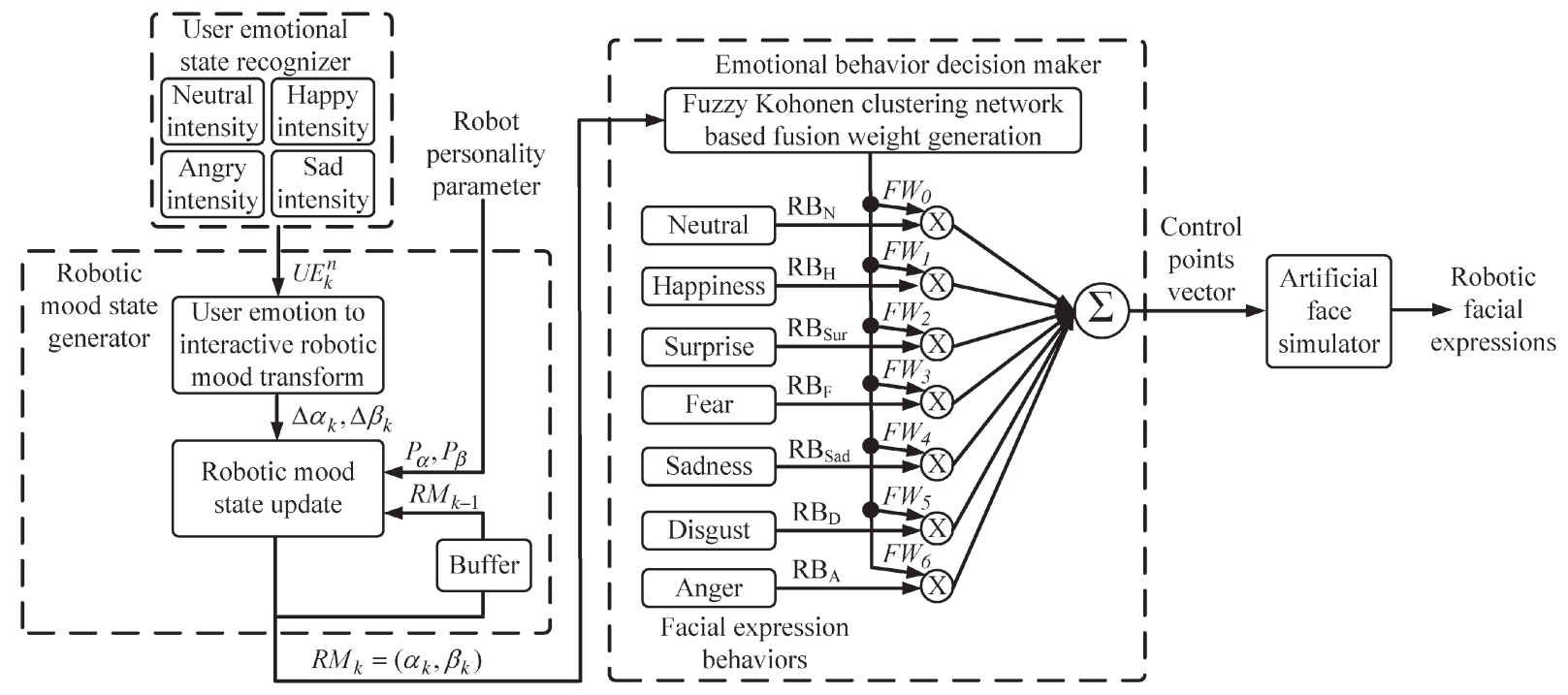

Fig. 1. Block diagram of the AEIS for an artificial face.

Previous related works show abundant powerful tools for designing emotional robots. It is observed that a proper mood state transition plays an important role in robotic emotional behavior generation. Robotic mood transition from the current to the next mood state directly influences the interaction behavior of the robot and also a user's feeling to the robot. However, most existing models treat mood transition by simple and intuitive representations. These representations lack a theoretical basis to support the assumptions in their mood state transition design. This motivated us to investigate a humanlike mood transition model for a robot by adopting well-studied mood state conversion criteria from psychological findings. The transition among mood states would become smoother and thus might enable a robot to respond with more natural emotional expressions. We further combine personality into the robotic mood model to represent the trait of the individual robot.

On the other hand, responsive interaction behaviors need to be designed to manifest the emotional intelligence of a robot. The relationship between mood states and responding behavior of a robot should not be a fixed one-to-one relation. A continuous robotic facial expression would be more interesting and natural to manifest the mood state transition. Instead of being arbitrarily defined, the relationships between robot emotional behaviors (e.g., in a form of facial expression) and mood state can be modeled from psychological analysis and utilized to build the interaction patterns in the design of expressive behaviors. Finally, in order to demonstrate the effectiveness of the proposed method, a 16-degree-of-freedom robotic head, as well as a comiclike face simulator [22], was utilized to demonstrate facial expressions generated by the proposed mood transition method. Questionnaire surveys were performed to examine the effectiveness of the design.

\section{Autonomous Emotional Interaction System}

Fig. 1 shows the block diagram of the proposed autonomous emotional interaction system (AEIS). Taking the robotic facial expression as an example, the robotic interaction is expected not only to react to the user's emotional state but also to reflect the mood transition of the robot itself. Responsive facial expressions should combine several basic facial expressions with varying emotional intensities. To do so, we attempt to integrate three modules to construct the AEIS, namely, user emotional state recognizer, robotic mood state generator, and emotional behavior decision maker. An artificial face is employed to demonstrate the effectiveness of the design. A camera is provided to capture the user's face in front of the robot. The acquired images are sent to the image processing stage for emotional state recognition [23]. The user emotional state recognizer is responsible for obtaining the user's emotional state and its intensity. In this design, the user's emotional state at instant $k\left(U E_{k}^{n}\right)$ is recognized and represented as a vector of four emotional intensities: neutral $\left(u e_{N, k}^{n}\right)$, happy $\left(u e_{H, k}^{n}\right)$, $\operatorname{angry}\left(u e_{A, k}^{n}\right)$, and $\operatorname{sad}\left(u e_{S, k}^{n}\right)$. Several existent emotional intensity estimation methods [24]-[27] provide effective tools to recognize the intensity of a human's emotional state. Their results can be applied and combined into the AEIS. In this paper, an image-based emotional intensity recognition module has been designed and implemented for the current design of AEIS. The recognized emotional intensity consists of basic emotional categories at each sampling instant and is represented by a value between 0 and 1 . These intensities are sent to the robotic mood state generator. Moreover, other emotion recognition modalities and methods (e.g., emotional speech recognition) can also be input to AEIS, and only the recognized emotional states contain intensity values between 0 and 1 .

In the robotic mood state generator, the recognized user's emotional intensities are transformed into interactive robotic mood variables represented by $\left(\Delta \alpha_{k}, \Delta \beta_{k}\right)$ (refer to Section III-A for detailed description). These two variables represent the way that a user's emotional state influences the robotic mood state transition. Furthermore, the robotic emotional behavior depends not only on the user's emotional state but also on the robot personality and previous mood state. Therefore, the proposed method takes into account the interactive robotic mood variables $\left(\Delta \alpha_{k}, \Delta \beta_{k}\right)$, previous robotic mood state $\left(R M_{k-1}\right)$, and robot personality parameters $\left(P_{\alpha}, P_{\beta}\right)$ to compute the current robotic mood state $\left(R M_{k}\right)$ 
(see Section III-D). In this paper, the current robotic mood state is represented as a point in the 2-D emotional plane. Furthermore, robotic personality parameters are created to describe the distinct humanlike personality of a robot. Based on the current robotic mood state, the emotional behavior decision unit autonomously generates suitable robot behavior in response to the user's emotion state.

For robotic emotional behavior generation, in response to the recognized user's emotional intensities, a set of fusion weights $\left(F W_{i}, i=0 \sim 6\right)$ corresponding to each basic emotional behavior is generated by using a fuzzy Kohonen clustering network (FKCN) (see Section IV) [28]. Similar to human beings, the facial expression of a robotic face is very complex and is difficult to be classified by a limited number of categories. In order to demonstrate interaction behaviors similar to that of humans, FKCN is adopted to generate an unlimited number of emotional expressions by fusing seven basic facial expressions. The outputs of FKCN are sent to the artificial face simulator to generate the interactive behaviors (facial expressions in this paper). An artificial face has been designed exploiting the method in [22] to demonstrate the facial expressions generated in human-robot interaction. Seven basic facial expressions are simulated, including neutral, happiness, surprise, fear, sadness, disgust, and anger. The facial expressions are depicted by moving control points determined from Ekman's model [29]. In the practical interaction scenario, each expression can be generated with different proportions of the seven basic facial expressions. The actual facial expression of the robot is generated by the summation of each behavior output multiplied by its corresponding fusion weight. Therefore, more subtle emotional expressions can be generated as desired. The detailed design of the proposed user emotional state recognition, robotic mood model, and interactive emotional behavior decision will be described in the following sections.

\section{Robotic Mood Transition Model}

Emotion is a complex psychological experience of an individual's state of mind as interacting with people or environmental influences. For humans, emotion involves "physiological arousal, expressive behaviors, and conscious experience" [30]. Emotional interaction behavior is associated with mood, temperament, personality, disposition, and motivation. In this paper, the emotion for robotic behavior is simplified to association with mood and personality. We apply the concept that emotional behavior is controlled by the current emotional state and mood, while the mood is influenced by personality. In this paper, a novel robotic mood state transition method is proposed for a given humanlike personality. Furthermore, the corresponding interaction behavior will be generated autonomously for a determined mood state.

\section{A. Responding to User's Emotional State}

A simple way to develop robotic emotional behaviors that can interact with people is to allow a robot to respond to emotional behaviors by mimicking humans. In human-robot emotional interaction, users' emotional expressions can be treated as trigger inputs to drive the robotic mood transition.
Furthermore, the transition of robotic mood depends not only on the user's emotional states but also on the robot mood and personality of itself. For a robot to interact with several individuals or a group of people, users' current (at instant $k$ ) emotional intensities $\left(U E_{k}^{n}\right)$ are sampled and transformed into interactive mood variables $\Delta \alpha_{k}$ and $\Delta \beta_{k}$ to represent how the user's emotional state influences the variation of robotic mood state transition.

From the experience of emotional interaction of human beings, a user's neutral intensity, for instance, usually affects the arousal and sleepiness mood variation directly. Thus, the robotic mood state tends to arousal while the user's neutral intensity is low. Similarly, the user's happiness, anger, and sadness intensities affect the pleasure-displeasure axes. Thus, the user's happy intensity will lead the robotic mood into pleasure. On the other hand, the robotic mood state behaves more displeasure while the user's angry and sad intensities are high. Based on the aforementioned observations, a straightforward case is designed for the interactive robotic mood variables $\left(\Delta \alpha_{k}, \Delta \beta_{k}\right)$, which represent the reaction from current users' emotional intensities on the pleasure-arousal plane, such that

$$
\begin{aligned}
\Delta \alpha_{k} & =\frac{1}{N_{s}} \sum_{n=1}^{N_{s}}\left[u e_{H, k}^{n}-\left(u e_{A, k}^{n}+u e_{S, k}^{n}\right) / 2\right] \\
\Delta \beta_{k}= & \frac{1}{N_{s}} \sum_{n=1}^{N_{s}} 2 \cdot\left(0.5-u e_{N, k}^{n}\right) \\
U E_{k}^{n}= & {\left[\begin{array}{l}
u e_{N, k}^{n} \\
u e_{H, k}^{n} \\
u e_{A, k}^{n} \\
u e_{S, k}^{n}
\end{array}\right] } \\
= & {\left[\begin{array}{ll}
k^{\mathrm{th}} & \text { neutral intensity for user } \mathrm{n} \\
k^{\text {th }} & \text { happiness intensity for user } \mathrm{n} \\
k^{\text {th }} & \text { anger intensity for user n } \\
k^{\text {th }} & \text { sadness intensity for user n }
\end{array}\right] }
\end{aligned}
$$

where $N_{\mathrm{s}}$ denotes the number of users and $U E_{k}^{n}$ represents four kinds of the $n$th user's emotional intensities. By using (1)-(3), the effect on robotic mood from multiple users' emotional inputs is represented. However, in this paper, only one user is considered for better concentration on the illustration of the proposed model, i.e., $N_{\mathrm{s}}=1$ in the following discussion. It is worth to extend the number of users in the next stage of this study such that a scenario like the Massachusetts Institute of Technology mood meter [31] can be investigated. Furthermore, the mapping between facial expressions of interacting human and robotic internal states may be modeled in a more sophisticated way. For example, $\Delta \alpha_{k}$ can be designed as $\left(u e_{A, k}^{i}+\right.$ $\left.u e_{S, k}^{i}\right) / 2-u e_{H, k}^{i}$ such that alternative (opposite) responses to a user can be obtained.

\section{B. Big Five Model}

McCrae et al. [32] proposed the Big Five factors (five factor model) to describe the traits of human personality. The Big Five model is an empirically based result, not a theory of personality. The Big Five factors were created through a statistical procedure, which was used to analyze how ratings of various personality traits are correlated for general humans. Table I 
TABLE I

Big Five Model of Personality

\begin{tabular}{ll}
\hline \hline Factor & \multicolumn{1}{c}{ Description } \\
\hline Openness & Open mindedness, interest in culture. \\
Conscientiousness & Organized, persistent in achieving goals. \\
Extraversion & Preference for and behavior in social situations. \\
Agreeableness & Interactions with others. \\
Neuroticism & Tendency to experience negative thoughts. \\
\hline \hline
\end{tabular}

lists the Big Five factors and their descriptions [33]. Moreover, Mehrabian [34] utilized the Big Five factors to represent the pleasure-arousability-dominance (PAD) temperament model. Through linear regression analysis, the scale of each PAD value is estimated by using the Big Five factors [35]. These results are summarized as three equations of temperament, which includes pleasure, arousability, and dominance.

In this paper, we adopted the Big Five model to represent the robot personality and determine the mood state transition on a 2-D pleasure-arousal plane. Hence, only two equations are utilized to represent the relationship between the robot personality and pleasure-arousal plane. Here, the elements of the Big Five factors are assigned based on a reasonable realization of Table I. Referring to [34], the robot personality parameters $\left(P_{\alpha}, P_{\beta}\right)$ are adopted such that

$$
\begin{aligned}
& P_{\alpha}=0.21 E+0.59 A+0.19 N \\
& P_{\beta}=0.15 O+0.3 A-0.57 N
\end{aligned}
$$

where $O, E, A$, and $N$ represent the Big Five factors of openness, extraversion, agreeableness, and neuroticism, respectively. Therefore, the robot personality parameters $\left(P_{\alpha}, P_{\beta}\right)$ are given as the robot personality is known, i.e., $O, E, A$, and $N$ are determined constants. Later, we will show that $\left(P_{\alpha}, P_{\beta}\right)$ works as the mood transition weightings on the pleasure ( $\alpha$ axis) and arousal ( $\beta$ axis) plane.

Note that the conscientiousness of the Big Five factors was not used in this design because this factor only influences the dominance axis of the 3-D PAD model. In this paper, the pleasure-arousal plane of the 2-D emotional model was applied, so only four out of five parameters are used to translate the mood transition weighting from the Big Five factors.

\section{Two-Dimensional Mood Space}

The relationship between mood states and emotional behaviors has been studied by psychologists. Russell et al. [36] proposed a 2-D scaling on the pleasure-displeasure and arousal-sleepiness axes to model the relationships between the facial expressions and mood state. In this paper, the result referenced from Russell et al. is employed to model the relationship between the mood state and output emotional behavior. Fig. 2 shows a 2-D scaling result for the general adult's facial expressions based on pleasure-displeasure and arousal-sleepiness ratings. As shown in Fig. 2, axes $\alpha$ and $\beta$ represent the amounts of pleasure and arousal, respectively. Eleven facial expressions are analyzed and located on the plane. The location of each facial expression is represented by a square along with its coordinates. The coordinates of each facial expression are obtained by measuring the location in the figure (interested readers are

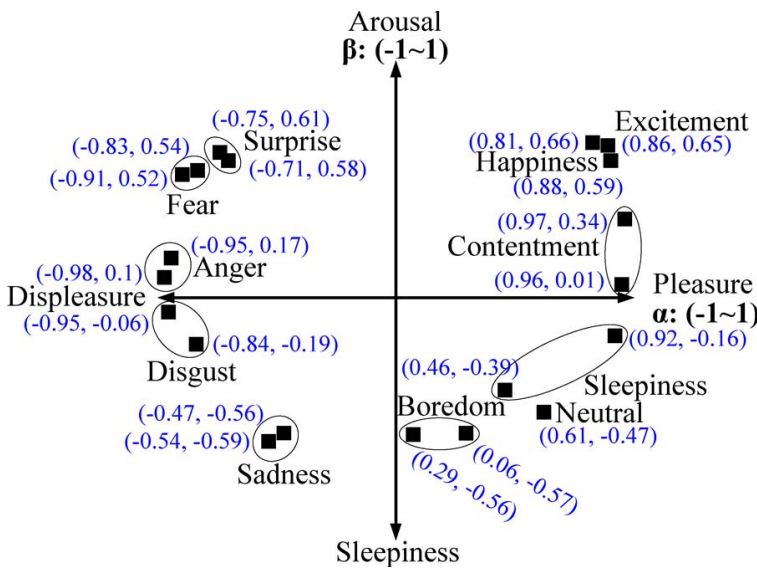

Fig. 2. Two-dimensional scaling for facial expressions based on pleasure-displeasure and arousal-sleepiness ratings.

referred to [36]). The relationship between robotic mood and output behavior, facial expression in this case, is determined.

\section{Robotic Mood State Generation}

As mentioned in Section III-A, both the user's current emotional intensity and robot personality affect the robotic mood transition. The way that robot personality affects the mood transition is described by robot personality parameters $\left(P_{\alpha}, P_{\beta}\right)$. As given in Section III-B, these two parameters act as weighting factors on the $\alpha$ and $\beta$ axes, respectively. When $P_{\alpha}$ and $P_{\beta}$ vary, the speed of mood transition in the direction of the $\alpha$ and $\beta$ axes is affected. On the other hand, the interactive mood variables $\left(\Delta \alpha_{k}, \Delta \beta_{k}\right)$ give the influence of the user's emotional intensity on the variation of robotic mood state transition. To reveal the relationship between robot personality and mood transition, we suggest to multiply robot personality parameters $\left(P_{\alpha}, P_{\beta}\right)$ with interactive mood variables $\left(\Delta \alpha_{k}, \Delta \beta_{k}\right)$. This indicates the influence of robotic mood transition from the current user's emotional intensity as well as robot personality.

Furthermore, the manifested emotional state is determined not only by the current robotic emotional variable but also by the previous robotic emotional states. The manifested robotic mood state at sample instant $k\left(R M_{k}\right)$ is calculated such that

$$
R M_{k} \equiv\left(\alpha_{k}, \beta_{k}\right)=R M_{k-1}+\left(P_{\alpha} \cdot \Delta \alpha_{k}, P_{\beta} \cdot \Delta \beta_{k}\right)
$$

where $\left(\alpha_{k}, \beta_{k}\right) \in[-1,1]$ represents the coordinates of the robotic mood state at sample instant $k$ on the pleasure-arousal plane. By using (6), the current robotic mood state is determined and located on the emotional plane. Moreover, the mood transition is influenced by personality, which is reflected by the Big Five factors. After obtaining the manifested robotic mood state $\left(R M_{k}\right)$, the coordinate of $\left(\alpha_{k}, \beta_{k}\right)$ will be mapped onto the pleasure-arousal plane, and a suitable corresponding facial expression can be determined, as shown in Fig. 2.

\section{INTERACTIVE EMOTIONAL BEHAVIOR GENERATION}

After the robotic mood state is determined by using (6), a suitable emotional behavior is expected to respond to the user. In this paper, we propose a design based on FKCN to generate 


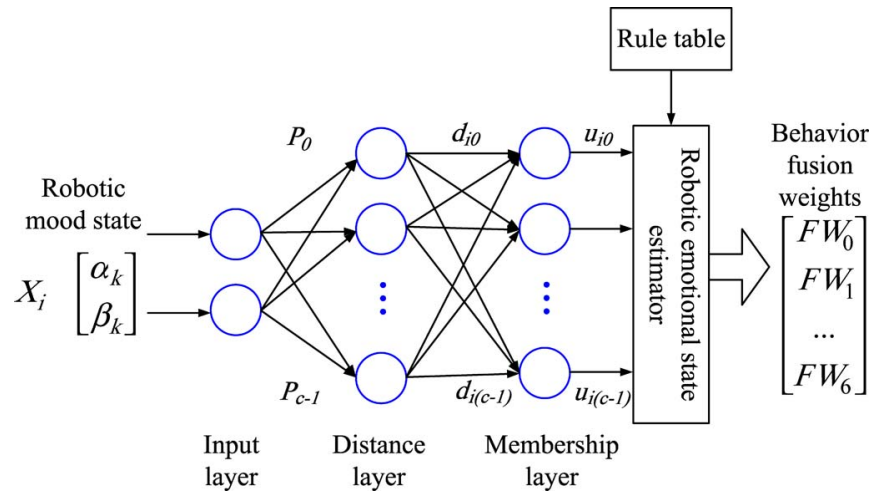

Fig. 3. Fuzzy-neuro network for fusion-weight generation.

smooth variation of interaction behaviors (facial expressions) as the mood state transits gradually.

\section{A. Proposed Expression-Fusion Design Based on FKCN}

In this approach, pattern recognition techniques were adopted to generate interactive robotic behaviors [21], [28]. By adopting FKCN, the robotic mood state, obtained from (6), is mapped to fusion weights of basic robotic emotional behaviors. The output will be a linear combination of weighted basic behaviors. In the current design, the basic facial expression behaviors are neutral, happiness, surprise, fear, sadness, disgust, and anger, as shown in Fig. 1. FKCN is employed to determine the fusion weight of each basic emotional behavior based on the current robotic mood. Fig. 3 shows the structure of the fuzzyneuro network for fusion-weight generation. In the input layer of the network, the robotic mood state $\left(\alpha_{k}, \beta_{k}\right)$ is regarded as inputs of FKCN. In the distance layer, the distance between the input pattern and each prototype pattern is calculated such that

$$
d_{i j}=\left\|X_{i}-P_{j}\right\|^{2}=\left(X_{i}-P_{j}\right)^{T}\left(X_{i}-P_{j}\right)
$$

where $X_{i}$ denotes the input pattern and $P_{j}$ denotes the $j$ th prototype pattern (see Section IV-B). In this layer, the degree of difference between the current robotic mood state and the prototype pattern is calculated. If the robotic mood state is not similar to the built-in prototype patterns, then the distance will reflect the dissimilarity. The membership layer is provided to map the distance $d_{i j}$ to membership values $u_{i j}$, and it calculates the similarity degree between the input pattern and the prototype patterns. If an input pattern does not match any prototype pattern, then the similarity between the input pattern and each individual prototype pattern is represented by a membership value from 0 to 1 . The determination of the membership value is given such that

$$
u_{i j}= \begin{cases}1 & \text { if } d_{i j}=0 \\ 0 & \text { if } d_{i k}=0(k>0, j \leq c-1)\end{cases}
$$

where $c$ denotes the number of prototype patterns; otherwise

$$
u_{i j}=\left[\sum_{l=0}^{c-1} \frac{d_{i j}}{d_{i l}}\right]^{-1} .
$$

Note that the sum of the outputs of the membership layer equals 1 . Using the rule table (see later) and the obtained membership values, the current fusion weights $\left(F W_{i}, i=0 \sim\right.$ 6 ) are determined such that

$$
F W_{i}=\sum_{j=0}^{c-1} w_{j i} u_{i j}
$$

where $w_{j i}$ represents the prototype-pattern weight of the $i$ th output behavior. The prototype-pattern weights are designed in a rule table to define the basic primitive emotional behaviors corresponding to carefully chosen input states.

\section{B. Rule Table}

In the current design, several representative input emotional states were selected from the 2-D model in Fig. 2, which gives the relationship between facial expressions and mood states. Each location of facial expression on the mood plane in Fig. 2 is used as a prototype pattern for FKCN. Thus, a rule table is constructed accordingly following the structure of FKCN. As shown in Table II, seven basic facial expressions were selected to build the rule table. The IF part of the rule table is the emotional state of $\alpha_{k}$ and $\beta_{k}$ of the pleasure-arousal space, and the THEN part is the prototype-pattern weight $\left(w_{j i}\right)$ of seven basic expressions. For example, the neutral expression in Fig. 2 occurs at $(0.61,-0.47)$, which forms the IF part of the first rule and the prototype pattern for neutral behavior. The THEN part of this rule is the neutral behavior expressed by a vector of prototype-pattern weights $(1,0,0,0,0,0,0)$. The other rules and prototype patterns are set up similarly following the values in Fig. 2. Some facial expressions are located at two distinct points on the mood space, both locations are employed, and two rules are set up following the analysis results from psychologists. There are altogether 13 rules as shown in Table II. Note that Table II gives us suitable rules to mimic the behavior of humans since the content of Fig. 2 is referenced from psychology results. However, other alternatives and more general rules can also be employed. FKCN works to generalize from these prototype patterns all possible situations (robotic mood state in this case) that may happen to the robot. In the FKCN generalization process, proper fusion weights for the corresponding pattern are calculated. After obtaining the fusion weights of output behaviors from FKCN, the robot's behavior is determined from seven basic facial expressions weighted by their corresponding fusion weights such that

$$
\begin{aligned}
& \text { Artifical facial expression } \\
& \begin{aligned}
= & \mathrm{RB}_{\mathrm{N}} \times F W_{0}+\mathrm{RB}_{\mathrm{H}} \times F W_{1}+\mathrm{RB}_{\mathrm{Sur}} \times F W_{2} \\
& +\mathrm{RB}_{\mathrm{F}} \times F W_{3}+\mathrm{RB}_{\mathrm{Sad}} \times F W_{4} \\
& +\mathrm{RB}_{\mathrm{D}} \times F W_{5}+\mathrm{RB}_{\mathrm{A}} \times F W_{6}
\end{aligned}
\end{aligned}
$$

where $\mathrm{RB}_{\mathrm{N}}, \mathrm{RB}_{\mathrm{H}}, \mathrm{RB}_{\mathrm{Sur}}, \mathrm{RB}_{\mathrm{F}}, \mathrm{RB}_{\mathrm{Sad}}, \mathrm{RB}_{\mathrm{D}}$, and $\mathrm{RB}_{\mathrm{A}}$ represent the behavior control vectors of neutral, happiness, surprise, fear, sadness, disgust, and anger, respectively. It is seen that (11) gives us a method to generate facial expressions by combining and weighting the seven basic expressions.

The linear combination of basic facial expressions gives a straightforward yet effective way to express various emotional 
TABLE II

Rule TABle For INTERACTIVE EMOTIONAL BEHAVIOR DECISION

\begin{tabular}{|c|c|c|c|c|c|c|c|c|c|}
\hline \multicolumn{3}{|c|}{$\begin{array}{c}\text { IF-part } \\
\text { prototype patterns }\end{array}$} & \multicolumn{7}{|c|}{ THEN-part weighting } \\
\hline$\overline{\# j}$ & $\alpha_{k}$ & $\beta_{k}$ & Neutral & Happiness & Surprise & Fear & Sadness & Disgust & Anger \\
\hline 1 & 0.61 & -0.47 & 1 & & & & & & \\
\hline 2 & 0.81 & 0.66 & & 1 & & & & & \\
\hline 3 & 0.88 & 0.59 & & 1 & & & & & \\
\hline 4 & -0.75 & 0.61 & & & 1 & & & & \\
\hline 5 & -0.71 & 0.58 & & & 1 & & & & \\
\hline 6 & -0.83 & 0.54 & & & & 1 & & & \\
\hline 7 & -0.91 & 0.52 & & & & 1 & & & \\
\hline 8 & -0.47 & -0.56 & & & & & 1 & & \\
\hline 9 & -0.54 & -0.59 & & & & & 1 & & \\
\hline 10 & -0.95 & -0.06 & & & & & & 1 & \\
\hline 11 & -0.84 & -0.19 & & & & & & 1 & \\
\hline 12 & -0.95 & 0.17 & & & & & & & 1 \\
\hline 13 & -0.98 & 0.1 & & & & & & & 1 \\
\hline
\end{tabular}

behaviors. In order to make the combined facial expression to be more consistent with human experience, an evaluation and adjusting procedure was carried out by a panel of students in the laboratory. The features of the seven basic facial expressions were adjusted as distinguished as possible to approach human perception experience. Some results of linear combination are demonstrated using a face expression simulator (please refer to Section IV-D).

In fact, human emotional expressions are difficult to be represented by a mathematical model or several typical rules. On the other hand, FKCN is very suitable for building up the emotional expressions. The merit of FKCN is its capacity to generalize the results using limited assigned rules (prototypes). Furthermore, dissimilar emotional types can be designed by adjusting the rules. For the artificial face, facial expressions are defined as the variation of control points, which are positions of the eyebrow, eye, lips, and wrinkles of the artificial face.

\section{Evaluation of FKCN Fusion-Weight Generation}

In order to verify the result of fusion-weight generation using FKCN, we applied the rules in Table II and simulated the results of weight distribution for various emotional states. The purpose is to evaluate how the proposed FKCN works to generalize any input emotional state $\left(\alpha_{k}, \beta_{k}\right)$ and gives a set of output fusion weights corresponding to the input. Fig. 4 shows a simulation result of the weight distribution of a basic expression versus robotic mood variation on the pleasure-arousal plane. Here, only one simulation output for neutral emotional expression is illustrated. The black squares in Fig. 4 indicate the robotic mood transition from $\left(\alpha_{k-1}, \beta_{k-1}\right)$ to $\left(\alpha_{k}, \beta_{k}\right)$. Fig. 4 shows the weight distribution of neutral expression for the whole robotic mood space. The same contour color in the figure has the identical neutral weight. The maximum weight (1) occurs at $(0.61,-0.47)$ in the pleasure-arousal plane. It is seen that the neutral weight decreases while the robotic mood state moves away from $(0.61,-0.47)$. These results coincide with the 2-D emotional state of facial expressions in Fig. 2. Furthermore, the correlation among seven basic emotional behaviors is also checked in the simulation. It is seen that a point on the mood

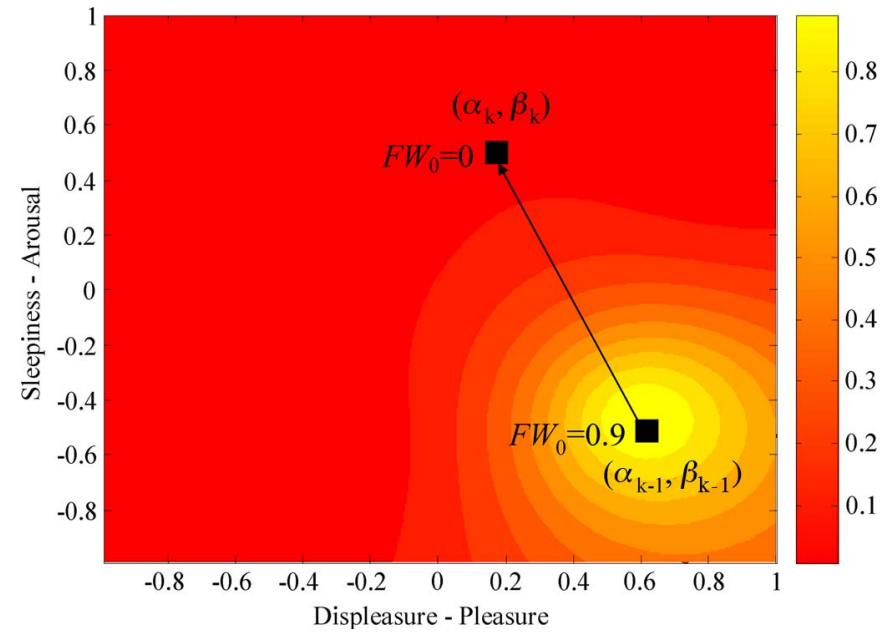

Fig. 4. Fusion-weight distribution for neutral facial expressions.

plane will map to a corresponding fusion weight for each of the seven basic emotional expressions.

\section{Animation of Artificial Face Simulator}

To evaluate the effectiveness of the FKCN-based behavior fusion on actual emotional expressions, we developed an artificial face simulator exploiting the method in [22] to examine robotic facial expressions. The artificial face illustrates the expression based on the contraction of facial muscles. It can also dynamically generate features such as wrinkles [22]. In this simulation, seven basic facial expressions, neutral, happiness, surprise, fear, sadness, disgust, and anger, are first designed by specifying the muscle tensions of each expression composed of seven different fusion weights. Table III shows some examples of the basic facial expressions generated by the simulator with different weights. One observes that the facial expression changes from smiling to laughing as the weight of happiness increases and from gloomy to crying as the weight of sadness increases. Finally, fused emotional expressions are depicted by the linear combination of weighted basic facial expressions. Table IV shows some examples of facial expressions generated by linear combination. 
TABLE III

Basic Facial EXPRESSions With VARIOUS Weights EXECUTED IN THE SiMULATOR

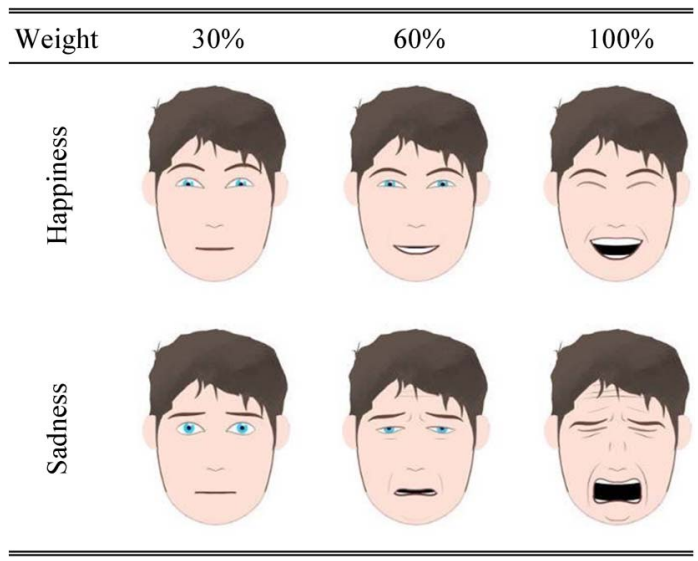

TABLE IV

LiNEAR COMBINED FACIAL EXPRESSIONS With VARIOUS WEIGHTS ON THE SIMULATOR

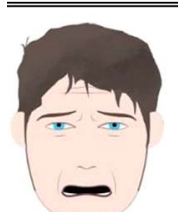

Sadness: $70 \%$

Anger: $30 \%$

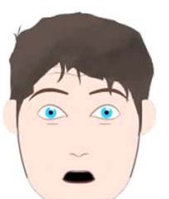

Suprise: $70 \%$ Anger:30\%

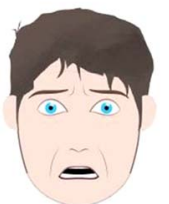

Fear:70\%

Sadness:30\%

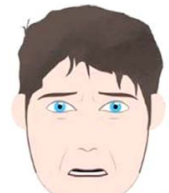

Sadness: $50 \%$ Anger: $50 \%$

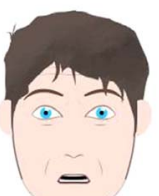

Suprise: $50 \%$ Anger:50\%

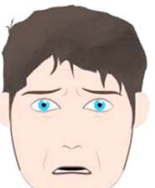

Fear: $50 \%$

Sadness: $50 \%$

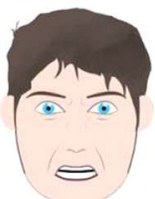

Sadness: $30 \%$ Anger:70\%

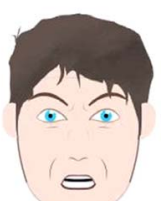

Suprise: $30 \%$ Anger:70\%

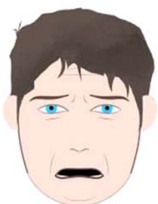

Fear: $30 \%$

Sadness: $70 \%$

\section{USER EMotional State ReCognition}

In this design, the user's emotional state, i.e., $U E_{k}^{n}$, is used as input to the system. In order to obtain $U E_{k}^{n}$, an imagebased facial expression recognition module has been designed and implemented. The facial expression recognition module consists of the face detection stage, feature extraction stage, and emotional intensity analyzer. The first step of the facial expression recognition module is to detect a human face in the acquired image frame. When an image frame is captured from the camera, skin color is utilized to segment possible human face areas in the image. The morphology closing procedure is applied to reduce the noise in the image frame. Then, human face candidates are obtained by using color region mapping techniques. Finally, the attentional cascade method [37] is used to determine which candidate is indeed a human face.

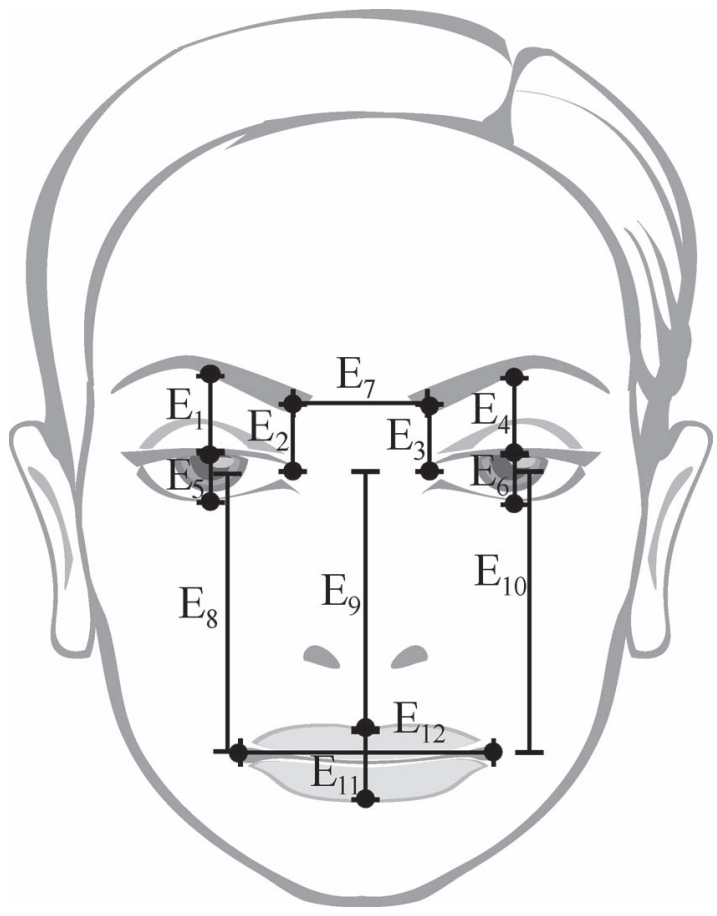

Fig. 5. Definition of the facial feature points and feature values.

After a face is detected and segmented, the feature extraction stage is employed to locate the eyes, eyebrows, and lip region in the human face area. The feature extraction module finds feature points from the detected frontal face image. Fig. 5 shows the definition of facial feature points and the feature values. Here, $E_{\mathrm{i}}(i=1 \sim 12)$ indicates the distance among the feature points. The system employs integral optical density (IOD) [38] to find the area of the eyes and eyebrows. IOD works on binary images and gives reliable position information of both eyes. In order to increase the robustness of feature point extraction, this method further combines the IOD result and edge features. Through an AND operation of two successive binary images, the contours of the eyes and eyebrows can be extracted. After obtaining the facial feature points, 12 significant feature values, which are distances between two selected feature points. In order to reduce the influence of distance between a user and the camera, these feature values are normalized for emotion recognition. Thus, every facial expression is presented as a feature set. For more detailed design steps of face detection and feature extraction processing, readers are referred to [39].

To recognize the user's emotional states, we further developed an image-based method to extract facial expression intensity. Four feature vectors, namely, $\vec{F}_{N e u}, \vec{F}_{H a}, \vec{F}_{A n g}$, and $\vec{F}_{S a d}$, are defined to represent the standard neutral, happy, angry, and sad expressions. Dissimilarities between the current feature set of a user $\left(\vec{F}_{U s e r, k}\right)$ and the standard facial expressions are calculated such that

$$
\begin{aligned}
d_{N, k} & =\left\|\vec{F}_{U s e r, k}-\vec{F}_{\text {Neu }}\right\| \\
d_{H, k} & =\left\|\vec{F}_{U s e r, k}-\vec{F}_{H a}\right\| \\
d_{A, k} & =\left\|\vec{F}_{U s e r, k}-\vec{F}_{A n g}\right\| \\
d_{S, k} & =\left\|\vec{F}_{U s e r, k}-\vec{F}_{S a d}\right\|
\end{aligned}
$$


TABLE V

Test Result of Emotion State Recognition

\begin{tabular}{|c|c|c|c|c|c|}
\hline Output & $\begin{array}{l}Z \\
\stackrel{Z}{\Xi} \\
ٍ\end{array}$ & 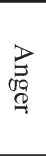 & 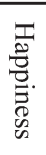 & $\begin{array}{l}\infty \\
\stackrel{0}{0} \\
\stackrel{\Xi}{0} \\
\infty \\
\infty\end{array}$ & 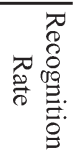 \\
\hline Neutral & 13 & 1 & 0 & 1 & $87 \%$ \\
\hline Anger & 0 & 15 & 0 & 0 & $100 \%$ \\
\hline Happiness & 2 & 0 & 13 & 0 & $87 \%$ \\
\hline Sadness & 1 & 1 & 0 & 13 & $87 \%$ \\
\hline
\end{tabular}

where $d_{N, K}, d_{H, K}, d_{A, K}$, and $d_{S, K}$ represent respectively the dissimilarities between the feature set of the user and the defined standard neutral, happy, angry, and sad expressions at sampling instant $k$. \|\| represents the Euclidean distance. In our design, the intensity of the user's emotion is recognized as the standard facial expression while the dissimilarities between the current feature set and standard facial expression are small. Therefore, the user's emotional intensities $U E_{k}^{n}$ are calculated such that

$$
\begin{aligned}
u e_{N, k}^{n} & =\frac{d_{N, k}^{-1}}{d_{N, k}^{-1}+d_{H, k}^{-1}+d_{A, k}^{-1}+d_{S, k}^{-1}} \\
u e_{H, k}^{n} & =\frac{d_{H, k}^{-1}}{d_{N, k}^{-1}+d_{H, k}^{-1}+d_{A, k}^{-1}+d_{S, k}^{-1}} \\
u e_{A, k}^{n} & =\frac{d_{A, k}^{-1}}{d_{N, k}^{-1}+d_{H, k}^{-1}+d_{A, k}^{-1}+d_{S, k}^{-1}} \\
u e_{S, k}^{n} & =\frac{d_{S, k}^{-1}}{d_{N, k}^{-1}+d_{H, k}^{-1}+d_{A, k}^{-1}+d_{S, k}^{-1}}
\end{aligned}
$$

where $u e_{N, k}^{n}, u e_{H, k}^{n}, u e_{A, k}^{n}$, and $u e_{S, k}^{n}$ represent respectively the $n$th user's emotional intensities at sampling instant $k$ for neutral, happy, angry, and sad expressions. By using this procedure, the user's emotional state is represented as a set of four emotional intensities.

In this paper, the Cohn-Kanade AU-Coded Facial Expression Database [40] is used to verify the proposed method of emotional state recognition. Twenty-four sets of facial images of different basic facial expressions were selected as training data. Each set contains seven facial images of a particular emotion with various facial expressions. Sixty face images of different basic facial expressions were selected as test data. To compare the system with the ground truth, we choose the strongest emotion as recognition results. The result of this experiment is shown in Table V. The average recognition rate is $90 \%$.

Fig. 6 shows an example of emotional state recognition. In this example, neutral, happy, angry, and sad facial expressions are used as testing samples. In Fig. 6(a), 14 dot marks represent the extracted feature points for facial expression recognition. The emotional intensities are obtained using (16)-(19). As shown in Fig. 6(a), the ratio of the neutral component amounts to $54 \%$, which dominates the facial expression, although the other emotion components also contribute to the facial expression. Similar results are obtained as shown in Fig. 6(b)-(d).

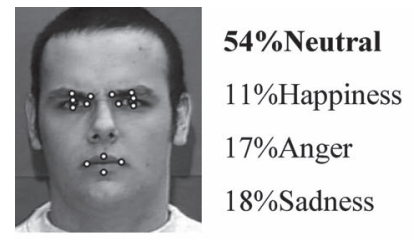

(a)

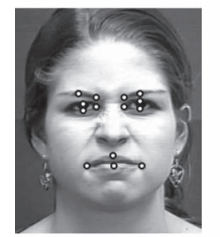

$15 \%$ Neutral $10 \%$ Happiness 57\%Anger $18 \%$ Sadness

(c)

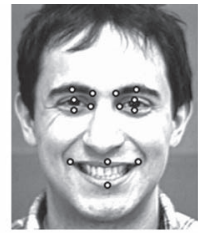

$15 \%$ Neutral 65\%Happiness $11 \%$ Anger 9\%Sadness

(b)

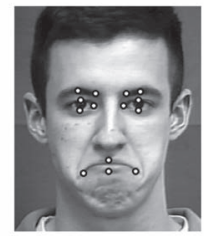

$8 \%$ Neutral 9\%Happiness $14 \%$ Anger $69 \%$ Sadness (d)

Fig. 6. Examples of user emotional state recognition.

\section{CMOS}

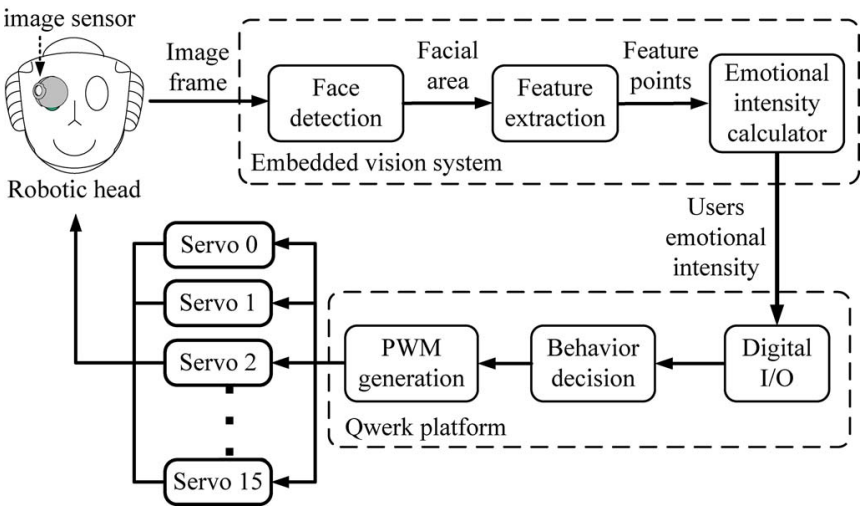

Fig. 7. Architecture of the self-built anthropomorphic robotic head.

\section{EXPERIMENTAL RESULTS}

The complete system has been tested and evaluated for autonomous emotional interaction. We first implemented the proposed AEIS on a self-constructed anthropomorphic robotic head for experimental validation. The robotic head, however, has some hardware limitations in completing the evaluation experiments of emotion transition system. The face simulator was adopted for testing the effectiveness of the proposed human-robot interaction design. Both results are presented hereinafter.

\section{A. Experiments on an Anthropomorphic Robotic Head}

In order to verify the developed algorithms for emotional human-robot interaction, an embedded robotic vision system [41] has been integrated with an anthropomorphic robotic head with 16 degrees of freedom. The DSP-based vision system was installed at the back of the robotic head, and the CMOS image sensor was put on the right eye to capture facial images. The system architecture of the robotic head is shown in Fig. 7. A Qwerk platform [42] works as an embedded controller. It receives the estimated emotional intensity of a user from the vision system and outputs the corresponding pulse width modulation signals to $16 R C$ servo to generate the corresponding robotic facial expression. Fig. 8 shows several basic facial expressions of the robotic head. 


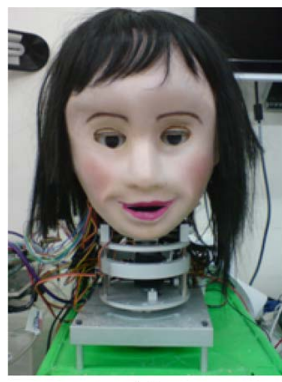

(a)

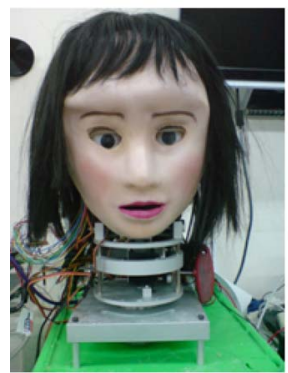

(d)

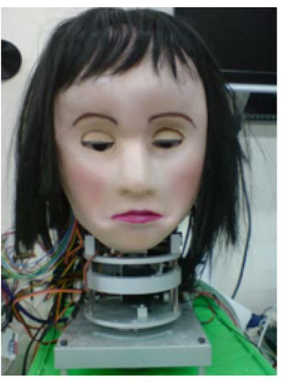

(b)

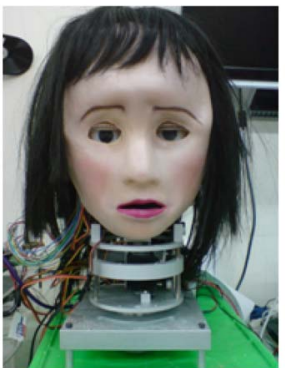

(e)

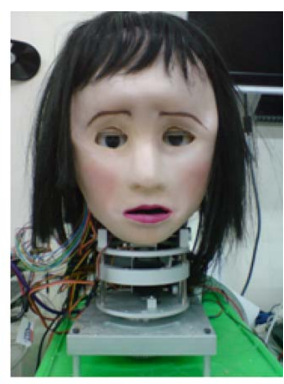

(c)

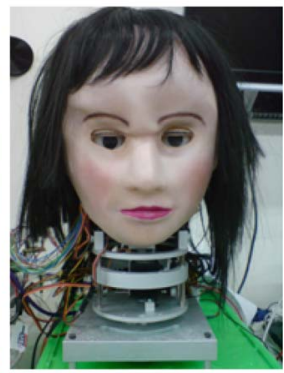

(f)
Fig. 8. Examples of facial expressions of the robotic head. (a) Happiness. (b) Disgust. (c) Sadness. (d) Surprise. (e) Fear. (f) Anger.

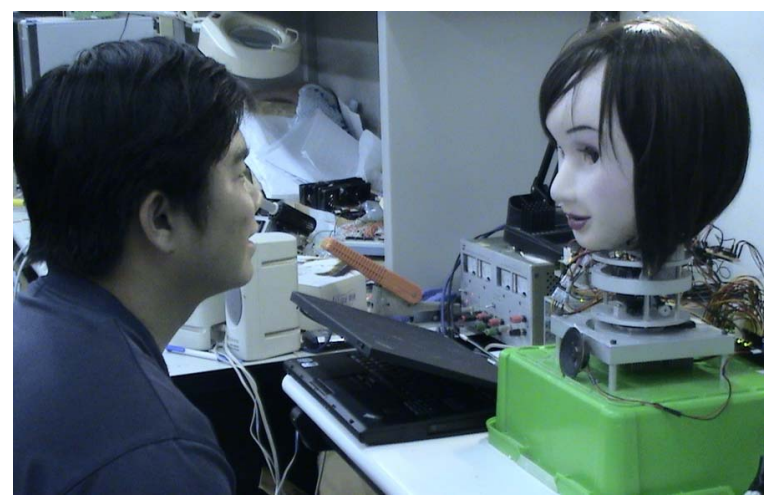

Fig. 9. Interaction scenario of a user and a robotic head.

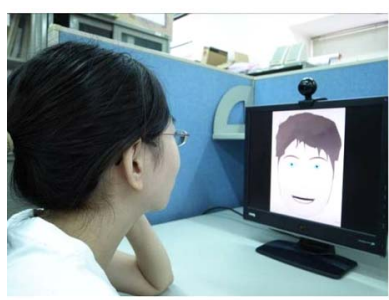

(a)

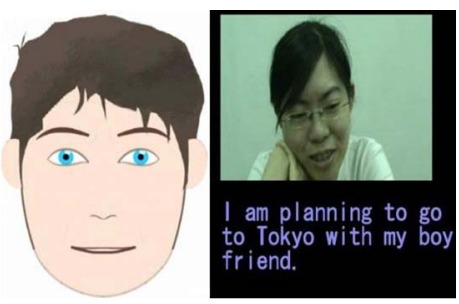

(b)
Fig. 10. Experiment setup: Interaction scenario with an artificial face.

In the experiment, a user presented his facial expressions in front of the robotic head as shown in Fig. 9. The robot responded to the user with different degrees of wondering as the user presented various intensities of surprise. A video clip of this experiment can be found in [43].

\section{B. Experimental Setup for the Artificial Face Simulator}

A virtual-conversation scenario was set up for testing the effectiveness of the proposed human-robot interaction design. As shown in Fig. 10(a), in the virtual-conversation test, a
TABLE VI

List of THE CONVERSATION DiAlogue AND CORRESPONDING SubJECT FACIAL EXPRESSIONS

\begin{tabular}{clc}
\hline $\begin{array}{c}\text { Sentence } \\
\#\end{array}$ & \multicolumn{1}{c}{ Dialogue } & $\begin{array}{c}\text { User's } \\
\text { emotional } \\
\text { state }\end{array}$ \\
\hline 1 & Hi, Robot. How are you feeling today? & Neutral \\
2 & I feel so bad today. I screwed up my job. & Sad \\
3 & $\begin{array}{l}\text { Do you know I feel very sad now? I really } \\
\text { hope it was not happened. }\end{array}$ & Sad \\
4 & $\begin{array}{l}\text { I am really angry at myself for my } \\
\text { mindless mistake. }\end{array}$ & Angry \\
5 & $\begin{array}{l}\text { However, in a few days it will be } \\
\text { Christmas. I think that I can get relaxed } \\
\text { during the vacation. }\end{array}$ & Neutral \\
6 & $\begin{array}{l}\text { I am planning to go to Tokyo with my } \\
\text { boyfriend. We hear of a carnival will take } \\
\text { place this year. }\end{array}$ & Happy \\
7 & Ha! I can't wait to go to the trip. & Happy \\
\hline \hline
\end{tabular}

TABLE VII

REGULATED USER EMOTION INTENSITY OF CONVERSION SENTENCES 1 AND 2

\begin{tabular}{cl} 
Sentence \# & $U E_{k}(k=1,2, \ldots, 15)$ \\
\hline \multirow{2}{*}{1} & $(0.5,0.2,0,0.3),(0.5,0.3,0,0.2),(0.5,0.4,0,0.1)$, \\
& $(0.6,0.4,0,0),(0.8,0.2,0,0),(1,0,0,0),(1,0,0,0)$. \\
& $(0.9,0,0,0.1),(0.8,0,0,0.2),(0.7,0,0,0.3),(0.6,0,0,0.4)$, \\
2 & $(0.5,0,0,0.5),(0.4,0,0,0.6),(0.4,0,0,0.6),(0.3,0,0,0.7)$. \\
\hline \hline
\end{tabular}

subject spoke to the artificial face (on the screen) while the talker's facial expression was detected by a web camera. The subject in the experiment is a student of the authors' institute. Table VI lists the conversation dialogue and corresponding subject facial expressions during the test. In the dialogue, the subject complained about her job with sad and angry facial expressions in the beginning. Then, the subject talked about the coming Christmas vacation. Her mood varied from an angry to a happy state. After acquiring facial images, the user emotional state recognizer transferred the user's facial expressions into sets of emotional intensity every $0.5 \mathrm{~s}$. The duration of this conversation is around $36 \mathrm{~s}$. There are 73 sets of emotional intensity values detected from the user in this conversation scenario. In order to observe the robotic emotional behavior purely due to individual personality and mood transition and avoid undesirable effect caused by error from user emotional state recognition, the detected user emotional intensities are regulated to more reasonable ones manually. Table VII shows part of the regulated user emotional intensities when the subject uttered sentences 1 and 2 . These sets of emotional intensity are utilized again as input to test the response of the artificial face with different robot personalities and moods.

\section{Evaluation of Robotic Mood Transition Due to Individual Personality}

It is desirable that a robot behaves differently in different interaction scenarios. For example, to keep attention from 
TABLE VIII

Definition of Personality Scales Using Big Five Factors

\begin{tabular}{ccc}
\hline \hline & $\begin{array}{c}\text { RobotA } \\
\text { (Active trait) }\end{array}$ & $\begin{array}{c}\text { RobotB } \\
\text { (Passive pessimist) }\end{array}$ \\
\hline Openness & 1 & 0.3 \\
Conscientiousness & 0.5 & 0.5 \\
Extraversion & 0.1 & 0.1 \\
Agreeableness & 0.5 & 0.2 \\
Neuroticism & 0.1 & 0.3 \\
$\left(P_{\alpha}, P_{\beta}\right)$ & $(0.34,0.24)$ & $(0.20,-0.07)$ \\
\hline \hline
\end{tabular}

students in education applications, the robot needs to behave more friendly and funny. Hence, the openness and agreeableness scales are designed higher. One can design the desired personality by adjusting the corresponding Big Five factors. In this experiment, two opposite robotic individual personalities were designed respectively for RobotA (with more active trait) and RobotB (with more passive trait). The Big Five factors were applied to model these two personalities. Table VIII lists the assigned scales corresponding to both opposite personalities. As we know, people belonging to the active trait are usually open-minded and interact with others more frequently. Hence, the openness and agreeableness scales of RobotA are higher than those of RobotB, and these two higher scales lead the personality parameters $\left(P_{\alpha}, P_{\beta}\right)$ to more positive tendency. Furthermore, a more passive pessimist has the tendency to experience negative thinking in general. Therefore, the neuroticism factor of RobotB is higher than that of RobotA. The higher neuroticism factor of RobotB leads its personality to more negative tendency on arousal ( $\beta$ axis). After trait values have been identified, the robot personality parameters $\left(P_{\alpha}, P_{\beta}\right)$ are determined by using (4) and (5). Moreover, the proposed robotic mood transition model is built accordingly.

To evaluate the effectiveness of the proposed emotional expression generation scheme based on individual personality, we conducted two sessions of experiments by using the artificial face as shown in Fig. 10(b). In the experiments, the same input sets were presented to RobotA and RobotB with the regulated user emotional intensities, respectively with the aforementioned conversation. The robotic mood states were observed as the same user spoke to RobotA and RobotB. Accordingly, the artificial face reacted with different facial expressions resulting from mood state transition. Video clips of this experimental can be found in [44].

Fig. 11 shows the mood transition of RobotA as the aforementioned conversation was performed. The initial mood state of RobotA was set at neutral state $(0.61,-0.47)$, referring to Fig. 2. The mood transition trajectories moved from the fourth quadrant to the third, the second, and the first quadrant in the end. The corresponding facial expressions varied from neutral (\#1) to boredom (\#2), sadness (\#3), anger (\#4), surprise (\#5), happiness (\#6), and excitement (\#7) in the end. The sharp turning point (\#5) in Fig. 11 indicates that RobotA recognized that the subject's emotional state varied rapidly from anger to happiness. Fig. 12 shows the mood transition of RobotB as the same emotional conversation was performed. The initial mood state of RobotB was also set on neutral state. The corresponding

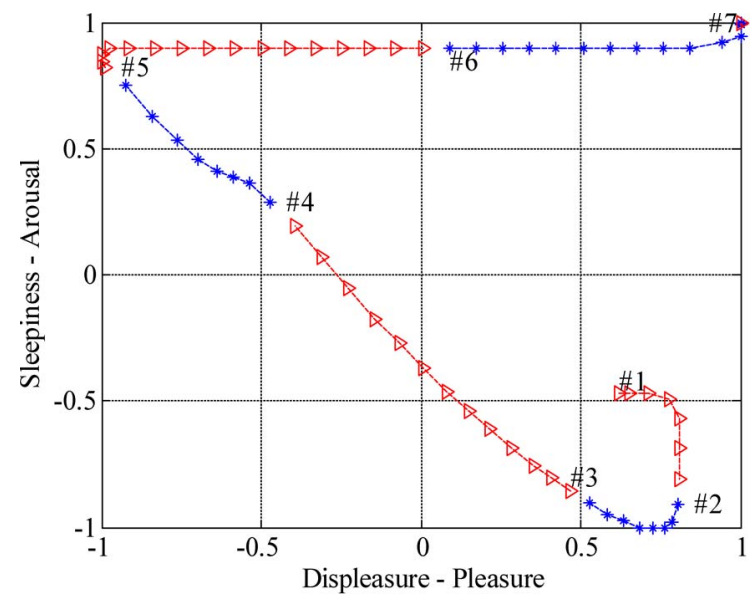

Fig. 11. Robotic mood transition of RobotA.

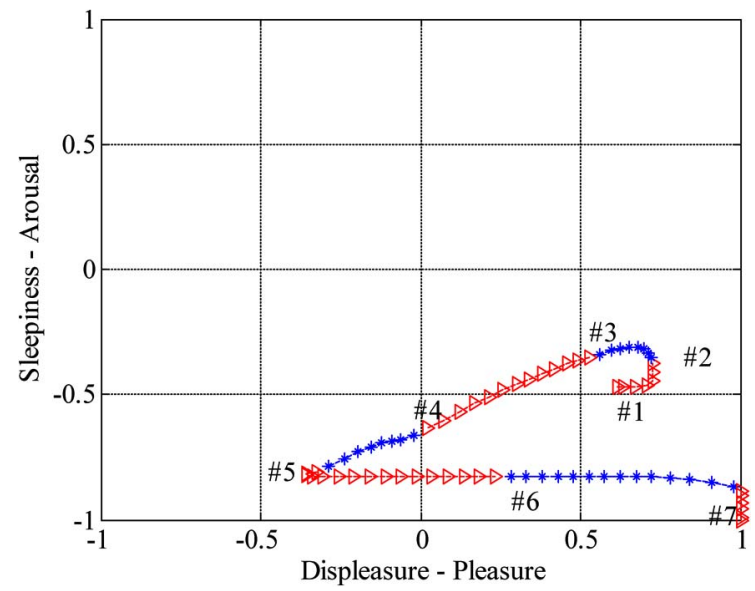

Fig. 12. Robotic mood transition of RobotB.

facial expressions varied from neutral (\#1) to sleepiness (\#2 and \#3), boredom (\#4), sadness (\#5), boredom (\#6), and then near neutral in the end. Compared with Fig. 11, the robotic mood transition of the passive trait is basically in the regions of boredom, sad, and neutral emotion. It stayed almost destructive no matter what kind of subject emotional states came into play. On the contrary, the robotic mood transition of the active trait scattered in the whole emotional space. These features manifest the difference in characters between the active and passive traits. This experiment reveals that the proposed mood transition scheme is able to realize robotic emotional behavior with different personality traits. Video clips of the mood transition for RobotA and RobotB can be found in [45].

Fig. 13 shows the variation of seven fusion weights while the subject uttered to RobotA. In the emotional conversation, the subject spoke seven dialogues as shown in Table VI. The corresponding fusion-weight variations of these seven dialogues are shown by seven sectors in Fig. 13. In dialogue \#1, the neutral facial expressions dominate the output behavior; this is reasonable since the subject's emotional state is neutral. In dialogues \#2 and \#3, the weights of sadness gradually increase while the transitions of the subject's emotional states are from neutral to sad. Next, the sad weight decreases, and the surprise weight increases as the subject feels angry progressively (dialogue \#4). 


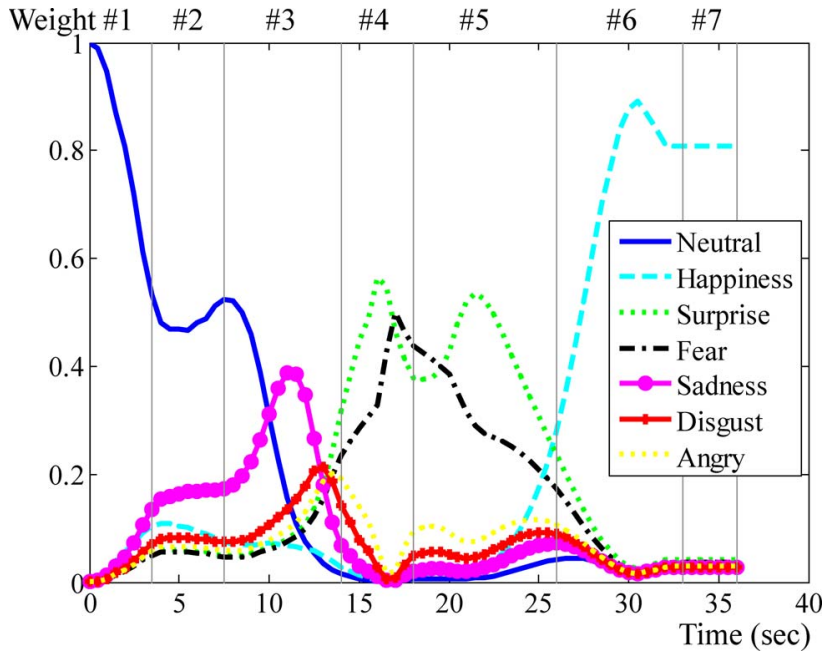

Fig. 13. Weight variation for RobotA (active trait).

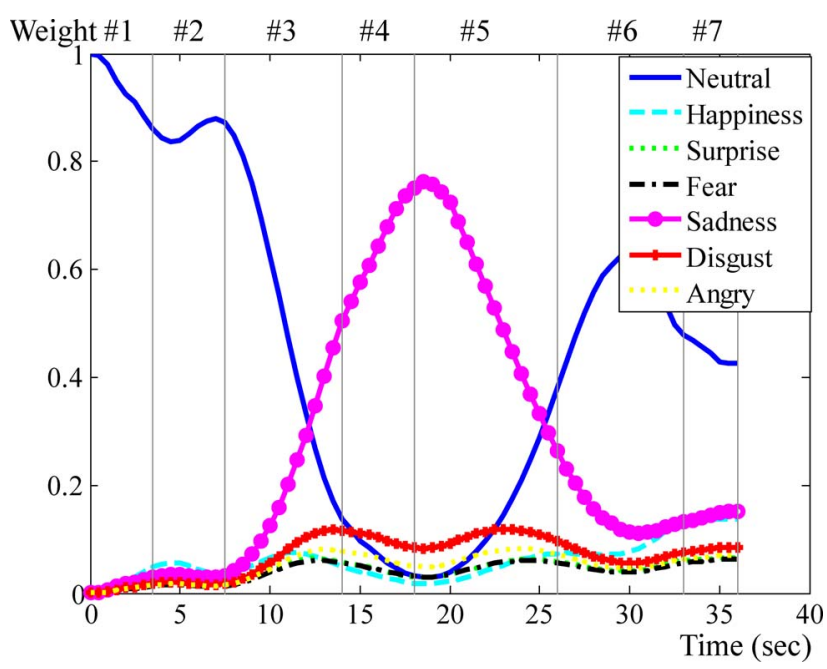

Fig. 14. Weight variation for RobotB (passive trait).

In the meantime, the fear weight also increases to respond to the subject's angry expression. After the subject turned to be happy, the surprise and fear weights decrease (dialogue \#5), and the happy weight increases to dominate the output behavior.

Fig. 14 shows the variation of the seven fusion weights as the subject uttered to RobotB with the same emotional conversation. In dialogues \#3 and \#4, the weights of sadness gradually increase while the transitions of the subject's emotional states are from neutral to sad and angry. After the subject's emotional states become happiness, the sad weight decreases (dialogue \#5), and the neutral weight increases to dominate the output behavior. Compared with RobotA in Fig. 13, the personality of the passive trait leads to less behavior variations and gets into the sadness emotion easily, although the subject's emotional states become happiness. These features match the emotional tendency for both the active and passive traits.

\section{Evaluation of Emotional Interaction Scheme}

In this experiment, questionnaire evaluation for the robot mood transition design was conducted for the emotional con-

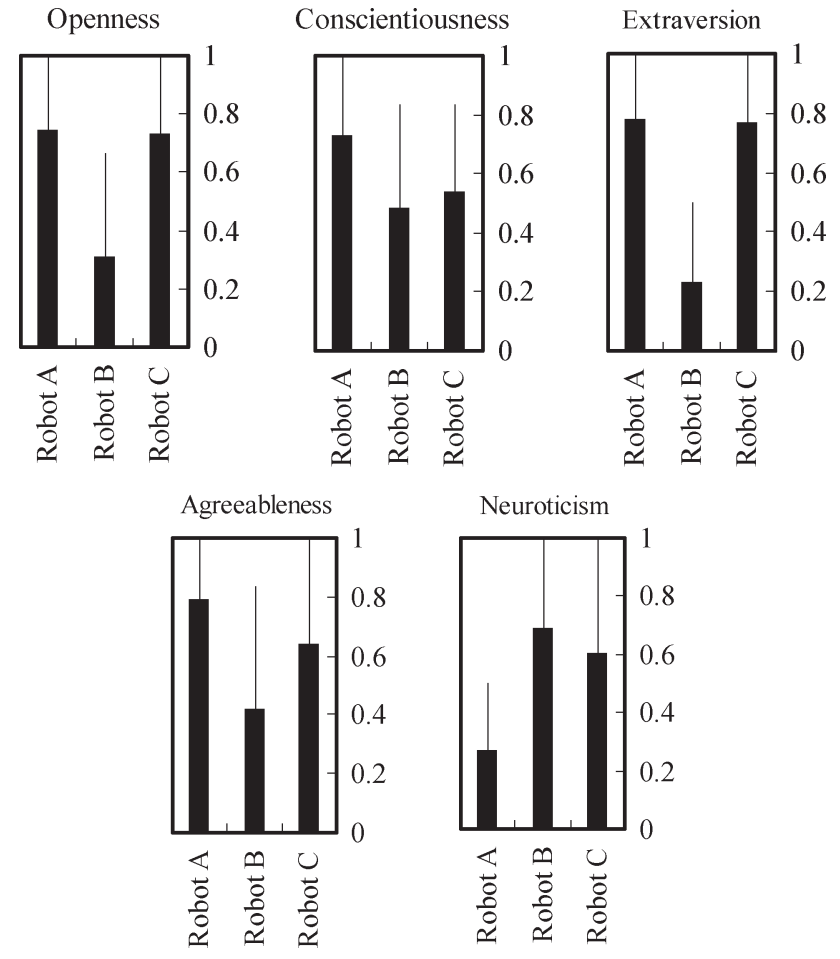

Fig. 15. Questionary result of psychological impact.

versation performed by the same subject with RobotA, RobotB, and RobotC, respectively. Here, the emotional response of RobotC was designed such that it is irrelevant to the proposed emotional interaction method. RobotC just follows facial expressions as recognized from the subject. The emotional conversation with RobotA, RobotB, and RobotC were recorded on three video clips [44] for questionnaire evaluation. We used the Big Five factors to evaluate the effectiveness of the proposed robotic emotional expression generation system.

Twenty subjects of age 20-40 were invited to watch the videos of virtual conversation with RobotA, RobotB, and RobotC. The invited subjects were asked to answer questionnaires after watching the aforementioned videos. In the questionnaire, a subject is asked to give scores from agreeing to disagreeing about the emotional interactions in the videos. We then average the scores using scales (0-1) for the RobotA, RobotB, and RobotC, respectively. The summary of the experimental results is shown in Fig. 15. In the current design, the facial expressions of the animation simulator are presented by the direct control of pure mood transition. Unlike wording wisdom of humans, the readability of facial expressions is related to very different underlying semantics [46]-[48]. Although the difference between the designed facial animation and human facial expression is obvious, the current design allows an observer to answer the questionnaires more straightforwardly. The major characteristics of the designed robotic trait (active and passive) are openness, agreeableness, and neuroticism. By observing the openness and agreeableness factors in Fig. 15, both factors are evaluated higher for RobotA than those of RobotB. It reveals that RobotA is recognized to have more tendencies to react and interact with humans than RobotB. Moreover, the neuroticism factor of RobotB is evaluated to be higher than that of RobotA. 


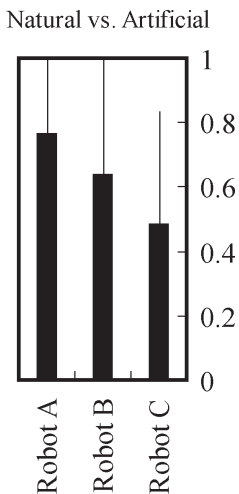

Fig. 16. Questionary result of natural versus artificial.

TABLE IX

Estimation OF PERSONALITY PARAMETERS BY QUESTIONNAIRE SURVEY

\begin{tabular}{ccc}
\hline \hline & RobotA & RobotB \\
\hline Openness & 0.74 & 0.31 \\
Conscientiousness & 0.73 & 0.48 \\
Extraversion & 0.78 & 0.23 \\
Agreeableness & 0.79 & 0.42 \\
Neuroticism & 0.27 & 0.69 \\
$\left(P_{\alpha}, P_{\beta}\right)$ & $(0.68,0.19)$ & $(0.43,-0.22)$ \\
\hline \hline
\end{tabular}

It indicates that the passive pessimist is indeed more inclined to experience negative thoughts than the active trait. These results conform to the designed personality in Table VIII.

As mentioned, RobotC only copies the subject's facial expressions without any mood transition discussed in this work. In other words, the detected Big Five factors of RobotC only show the subject's personality. In order to verify the difference between robots with the proposed mood transition scheme (RobotA and RobotB) and without it (RobotC), the same 20 subjects answered the questionnaire after watching the videos in [44]. In the questionnaire, a subject is asked to give scores from agreeing to disagreeing about the degree of natural or artificial interactions in the videos. The summary of the experimental results is shown in Fig. 16. Based on the item of natural versus artificial in Fig. 16, RobotA and RobotB both behave more naturally than RobotC. It shows that the proposed mood transition method enables the robot to behave in a humanlike manner.

Table IX shows the average values of 20 questionnaire surveys. The personality parameters of RobotA and RobotB are estimated as $(0.68,0.19)$ and $(0.43,-0.22)$, respectively. By comparing with the designed personality in Table VIII, we see that the personality parameters of RobotA and RobotB are $(0.34,0.24)$ and $(0.20,-0.07)$, respectively. It is seen that both $P_{\alpha}$ values $(0.34$ and 0.20$)$ of the designed RobotA and RobotB are proportional to the estimated $P_{\alpha}$ values $(0.68$ and 0.43 ) in Table IX, respectively. It reveals that both the designed and estimated mood transition velocities of RobotA are about 1.6 times $(0.68 / 0.43$ and $0.34 / 0.20)$ those of RobotB on the pleasure-displeasure axes. In another word, both the designed and the estimated RobotA are happier easily than RobotB with a similar ratio. Furthermore, both of the designed and estimated $P_{\beta}$ values of RobotB are negative. It indicates that both the designed and the estimated RobotA will tend to arousal and RobotB will tend to sleepiness while the same user's emotional intensity is imported. Hence, the estimated results of robot personality parameters are consistent with the designed personality scales in Table VIII. Based on the experimental results, it can be concluded that a robot can be designed with a desired personality and differently designed robotic personalities give distinct interactive behaviors. Moreover, the emotional robots behave more humanlike interaction.

\section{CONCLUSION}

A method of robotic mood transition for autonomous emotional interaction has been developed. An emotional model is proposed for mood state transition exploiting a robotic personality approach. Via adopting the psychological Big Five factors in the 2-D emotional model, the proposed method generates facial expressions in a more natural manner. The FKCN architecture, together with rule tables from psychological findings, sufficiently provides behavior fusion capability for a robot to generate emotional interactions. Experimental results reveal that the simulated artificial face interacts with people in a manner of mood transition and with robotic personality. The questionnaire investigation confirms positive results on the evaluation of responsive robotic facial expressions generated by the proposed design. In the future, more comparisons with other emotional models will be further studied. We will also investigate different models for robotic emotion generation and evaluate their emotional intelligence with practical experiments.

\section{ACKNOWLEDGMENT}

The authors would like to thank F.-H. Jen, J.-C. Tai, D.-H. Liang, and W.-J. He of the Minghsin University of Science and Technology, Taiwan, for their assistance in developing the robotic head.

\section{REFERENCES}

[1] C. Breazeal, "Emotion and sociable humanoid robots," Int. J. HumanComput. Studies, vol. 59, no. 1/2, pp. 119-155, Jul. 2003.

[2] C. Breazeal, D. Buchsbaum, J. Gray, D. Gatenby, and B. Blumberg, "Learning from and about others: Towards using imitation to bootstrap the social understanding of others by robots," J. Artif. Life, vol. 11, no. 1/2, pp. 1-32, Jan. 2005.

[3] MIT Media Lab, Personal Robot Group, Cambridge, MA. [Online]. Available: http://robotic.media.mit.edu/projects/robots/mds/headface/ headface.html

[4] T. Wu, N. J. Butko, P. Ruvulo, M. S. Bartlett, and J. R. Movellan, "Learning to make facial expressions," in Proc. IEEE 8th Int. Conf. Develop. Learn., Shanghai, China, 2009, pp. 1-6.

[5] N. Mavridis and D. Hanson, "The IbnSina Center: An augmented reality theater with intelligent robotic and virtual characters," in Proc. IEEE 18th Int. Symp. Robot Human Interactive Commun., Toyama, Japan, 2009, pp. 681-686.

[6] N. Mavridis, A. AlDhaheri, L. AlDhaheri, M. Khanji, and N. AlDarmaki, "Transforming IbnSina into an advanced multilingual interactive android robot," in Proc. IEEE GCC Conf. Exhib., Dubai, United Arab Emirates, 2011, pp. 120-123.

[7] T. Hashimoto, S. Hiramatsu, T. Tsuji, and H. Kobayashi, "Realization and evaluation of realistic nod with receptionist robot SAYA," in Proc. IEEE 
16th Int. Symp. RO-MAN Interactive Commun., Jeju Island, Korea, 2007, pp. 326-331.

[8] T. Hashimoto, S. Hiramatsu, T. Tsuji, and H. Kobayashi, "Development of the face robot SAYA for rich facial expressions," in Proc. Int. Joint Conf. SICE-ICASE, Busan, Korea, 2006, pp. 5423-5428.

[9] D. W. Lee, T. G. Lee, B. So, M. Choi, E. C. Shin, K. W. Yang, M. H. Back, H. S. Kim, and H. G. Lee, "Development of an android for emotional expression and human interaction," in Proc. Int. Fed. Autom. Control, Seoul, Korea, 2008, pp. 4336-4337.

[10] M. S. Siegel, "Persuasive Robotics: How Robots Change Our Minds," M.S. thesis, Massachusetts Inst. of Technol., Cambridge, MA, 2009.

[11] N. Mavridis, M. Petychakis, A. Tsamakos, P. Toulis, S. Emami, W. Kazmi, C. Datta, C. BenAbdelkader, and A. Tanoto, "FaceBots: Steps towards enhanced long-term human-robot interaction by utilizing and publishing online social information," Springer Paladyn J. Behav. Robot., vol. 1, no. 3, pp. 169-178, Sep. 2011.

[12] H. Miwa, T. Okuchi, K. Itoh, H. Takanobu, and A. Takanishi, "A new mental model for humanoid robots for human friendly communication introduction of learning system, mood vector and second order equations of emotion," in Proc. IEEE Int. Conf. Robot. Autom., Taipei, Taiwan, 2003, pp. 3588-3593.

[13] H. Miwa, K. Itoh, M. Matsumoto, M. Zecca, H. Takanobu, S. Rocella, M. C. Carrozza, P. Dario, and A. Takanishi, "Effective emotional expressions with expression humanoid robot WE-4RII: Integration of humanoid robot hand RCH-1," in Proc. IEEE/RSJ Int. Conf. Intell. Robots Syst., Sendai, Japan, 2004, pp. 2203-2208.

[14] D. Duhaut, "A generic architecture for emotion and personality," in Proc. IEEE Int. Conf. Adv. Intell. Mechatron., Xian, China, 2008, pp. 188-193.

[15] L. Moshkina, S. Park, R. C. Arkin, J. K. Lee, and H. Jung, "TAME: Timevarying affective response for humanoid robots," Int. J. Social Robot., vol. 3, no. 3, pp. 207-221, 2011.

[16] C. Itoh, S. Kato, and H. Itoh, "Mood-transition-based emotion generation model for the robot's personality," in Proc. IEEE Int. Conf. Syst., Man Cybern., San Antonio, TX, 2009, pp. 2957-2883.

[17] S. C. Banik, K. Watanabe, M. K. Habib, and K. Izumi, "An emotionbased task sharing approach for a cooperative multiagent robotic system," in Proc. IEEE Int. Conf. Mechatron. Autom., Kagawa, Japan, 2008, pp. $77-82$.

[18] J. C. Park, H. R. Kim, Y. M. Kim, and D. S. Kwon, "Robot's individual emotion generation model and action coloring according to the robot's personality," in Proc. IEEE Int. Symp. Robot Human Interactive Commun., Toyama, Japan, 2009, pp. 257-262.

[19] H. R. Kim and D. S. Kwon, "Computational model of emotion generation for human-robot interaction based on the cognitive appraisal theory," Int. J. Intell. Robot. Syst., vol. 60, no. 2, pp. 263-283, Nov. 2010.

[20] D. Lee, H. S. Ahn, and J. Y. Choi, "A general behavior generation module for emotional robots using unit behavior combination method," in Proc. IEEE Int. Symp. Robot Human Interactive Commun., Toyama, Japan, 2009, pp. 375-380.

[21] M. J. Han, C. H. Lin, and K. T. Song, "Autonomous emotional expression generation of a robotic face," in Proc. IEEE Int. Conf. Syst., Man Cybern., San Antonio, TX, 2009, pp. 2427-2432.

[22] Grimace Project. [Online]. Available: http://grimace-project.net/

[23] K. T. Song, M. J. Han, and J. W. Hong, "Online learning design of an image-based facial expression recognition system," Intell. Service Robot., vol. 3, no. 3, pp. 151-162, Jul. 2010.

[24] M. A. Amin and H. Yan, "Expression intensity measurement from facial images by self organizing maps," in Proc. Int. Conf. Mach. Learn. Cybern., Kunming, China, 2008, pp. 3490-3496.

[25] M. Beszedes and P. Culverhouse, "Comparison of human and automatic facial emotions and emotion intensity levels recognition," in Proc. Int. Symp. Image Signal Process. Anal., Istanbul, Turkey, 2007, pp. 429-434.

[26] M. Oda and K. Isono, "Effects of time function and expression speed on the intensity and realism of facial expressions," in Proc. IEEE Int. Conf. Syst., Man Cybern., Singapore, 2008, pp. 1103-1109.

[27] K. K. Lee and Y. Xu, "Real-time estimation of facial expression intensity," in Proc. IEEE Int. Conf. Robot. Autom., Taipei, Taiwan, 2003, pp. 2567-2572.

[28] K. T. Song and J. Y. Lin, "Behavior fusion of robot navigation using a fuzzy neural network," in Proc. IEEE Int. Conf. Syst., Man Cybern., Taipei, Taiwan, 2006, pp. 4910-4915.

[29] P. Ekman and W. V. Friesen, The Facial Action Coding System: A Technique for The Measurement of Facial Movement. Palo Alto, CA: Consulting Psychologists Press, 1978.

[30] D. G. Myers, Theories of Emotion. New York: Worth Publishers, 2004.
[31] MIT Meter Measures the Mood of Passers-By. [Online]. Available: http://www.popsci.com/technology/article/2011-11/mit-meter-measuresmood-passers

[32] R. R. McCrae and P. T. Costa, "Validation of the five-factor model of personality across instruments and observers," J. Personality Social Psychol., vol. 51, no. 1, pp. 81-90, Jan. 1987.

[33] P. T. Costa and R. R. McCrae, "Normal personality assessment in clinical practice: The NEO personality inventory," J. Psychol. Assess., vol. 4, no. 1, pp. 5-13, Mar. 1992.

[34] A. Mehrabian, "Analysis of the big-five personality factors in terms of the PAD temperament model," Australian J. Psychol., vol. 48, no. 2, pp. 8692, Aug. 1996.

[35] L. R. Goldberg, "The development of markers for the big-five factor structure," Psychol. Assess., vol. 4, no. 1, pp. 26-42, Mar. 1992.

[36] J. A. Russell and M. Bullock, "Multidimensional scaling of emotional facial expressions: Similarity from preschoolers to adults," J. Personality Social Psychol., vol. 48, no. 5, pp. 1290-1298, May 1985.

[37] P. Viola and M. Jones, "Rapid object detection using a boosted cascade of simple features," in Proc. IEEE Int. Conf. Comput. Vis. Pattern Recognit., Kauai, HI, 2001, pp. 511-518.

[38] J. H. Lai, P. C. Yuen, W. S. Chen, S. Lao, and M. Kawade, "Robust facial feature point detection under nonlinear illuminations," in Proc. IEEE ICCV Workshop Recognit., Anal. Tracking Faces Gestures Real-time Syst., Vancouver, Canada, 2001, pp. 168-174.

[39] M. J. Han, J. H. Hsu, K. T. Song, and F. Y. Chang, "A new information fusion method for bimodal robotic emotion recognition," J. Comput., vol. 3, no. 7, pp. 39-47, Jul. 2008.

[40] Cohn-Kanade AU-Coded Facial Expression Database. [Online]. Available: http://www.pitt.edu/ jeffcohn/CKandCK+.htm

[41] H. Andrian and K. T. Song, "Embedded CMOS imaging system for realtime robotic vision," in Proc. IEEE/RSJ Int. Conf. Intell. Robots Syst., Edmonton, Canada, 2005, pp. 3694-3699.

[42] Qwerk, Charmed Labs. [Online]. Available: http://www.charmedlabs. com/index.php?option=com_content\&amp;task=view\&amp;id=29

[43] [Online]. Available: http://isci.cn.nctu.edu.tw/video/ AnthropomorphicRobot/

[44] [Online]. Available: RoboticMoodTransition/

[45] [Online]. Available: RoboticMoodTransitionAnalysis/

http://isci.cn.nctu.edu.tw/video/ http://isci.cn.nctu.edu.tw/video/

[46] M. E. Hoque, R. E. Kaliouby, and R. W. Picard, "When human coders (and machines) disagree on the meaning of facial affect in spontaneous videos," in Proc. 9th Int. Conf. Intell. Virtual Agents, Amsterdam, Netherlands, 2009, pp. 337-343.

[47] M. E. Hoque, L.-P. Morency, and R. W. Picard, "Are you friendly or just polite?-Analysis of smiles in spontaneous face-to-face interactions," in Proc. 4th Int. Conf. Affective Comput. Intell. Interact., Memphis, TN, 2011, pp. 135-144.

[48] M. E. Hoque and R. W. Picard, "Acted vs. natural frustration and delight: Many people smile in natural frustration," in Proc. IEEE 9th Int. Conf. Autom. Face Gesture Recognit., Santa Barbara, CA, 2011, pp. 354-359.

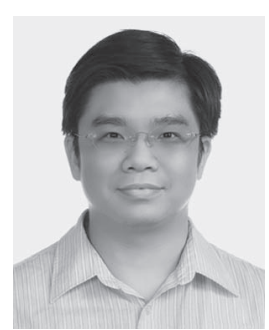

Meng-Ju Han received the B.S. degree in electrical engineering from the National Taipei University of Technology, Taipei, Taiwan, in 1998 and the M.S. degree in electrical engineering from the National Chung Hsing University, Taichung, Taiwan, in 2003. $\mathrm{He}$ is working toward the Ph.D. degree in electrical and computer engineering at National Chiao Tung University.

Since 2010, he has been an Associate Researcher with the Mechanical and Systems Research Laboratories, Industrial Technology Research Institute, Hsinchu, Taiwan. His research interests include human-robot interaction, emotion recognition, image processing, and visual tracking. 


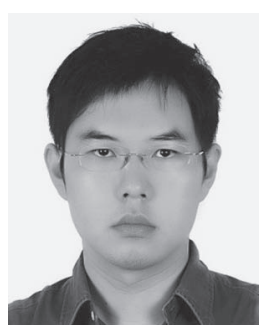

Chia-How Lin received the B.S and M.S. degrees in electrical and control engineering from the National Chiao Tung University, Hsinchu, Taiwan, in 2001 and 2003, respectively, where he is currently working toward the Ph.D. degree in electrical and computer engineering.

His areas of research interests include multiagent system, robot control system, sensor network, computer vision, and image-based robot navigation.

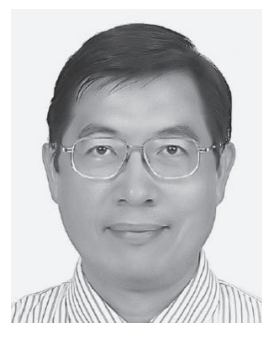

Kai-Tai Song (A'91-M'09) received the B.S. degree in power mechanical engineering from National Tsing Hua University, Hsinchu, Taiwan, in 1979 and the $\mathrm{Ph} . \mathrm{D}$. degree in mechanical engineering from the Katholieke Universiteit Leuven, Leuven, Belgium, in 1989.

Since 1989, he has been on the faculty and is currently a Professor with the Department of Electrical and Computer Engineering and the Institute of Electrical Control Engineering, National Chiao Tung University (NCTU), Hsinchu. He served as the Director of the Institute of Electrical Control Engineering from 2009 to 2011 and the Associate Dean of the Office of Research and Development of NCTU from 2007 to 2009. His areas of research interest include mobile robots, image processing, visual tracking, mobile manipulation, embedded systems, and mechatronics.

Dr. Song received the excellent award in automatic control engineering of the Chinese Automatic Control Society in 2010. He served as the Chairman of the IEEE Robotics and Automation Chapter, Taipei Section, in 1999. He served as the Program Chair of the 8th Asian Control Conference (ASCC 2011). 